\title{
Surface Area and Conductivity of Open-Cell Carbon Foams
}

\author{
Adriana M. Druma, M. Khairul Alam*, Calin Druma \\ Department of Mechanical Engineering, Ohio University \\ Athens, OH 45701 \\ *Correspondence Author's Email: alam@ohio.edu
}

\begin{abstract}
High thermal conductivity carbon foams have recently emerged as an effective thermal management material for space applications due to their lightweight. Open cell carbon foams are generally processed from pitch material obtained from coal or petroleum. These foams have spherical pores that create a three dimensional network of ligaments and nodes of complex geometry. The thermal conductivity of carbon foams can be studied numerically by finite element method; however the analysis requires a very fine grid that captures the microstructure of the foam. In this work, an analytical model for surface area and thermal conductivity is developed for a foam. To reduce the computational effort, an electrical circuit network analogy is used to calculate the bulk thermal conductivity of the foam. The analytical solution is then compared with semiempirical models, FEM solution and other analytical solutions.
\end{abstract}

\section{Nomenclature}

A total cross-sectional area of the unit cell $\left[\mathrm{m}^{2}\right]$;

$A_{g}$ cross-sectional area of the gas phase in the unit cell $\left[\mathrm{m}^{2}\right]$;

$A_{s}$ cross-sectional area of the solid phase in the unit cell $\left[\mathrm{m}^{2}\right]$;

$K_{e}$ effective thermal conductivity [W/mK];

$K_{g}$ gas thermal conductivity $[\mathrm{W} / \mathrm{mK}]$;

$K_{s}$ solid thermal conductivity [W/mK];

$K_{s j}$ solid thermal conductivity (at the juncture or nodes) $[\mathrm{W} / \mathrm{mK}]$;

$K_{s l}$ solid thermal conductivity in the ligaments (longitudinal direction) $[\mathrm{W} / \mathrm{mK}]$;

$K_{s t}$ solid thermal conductivity in the ligaments (transversal direction) $[\mathrm{W} / \mathrm{mK}]$;

$P$ porosity [\%];

$R_{p}$ radius of the pores [m];

$R_{t}$ is the thermal resistance of the foam $\left[{ }^{\circ} \mathrm{C} / \mathrm{W}\right]$.

$D_{p}$ pore diameter [m];

$F$ is the solid conduction efficiency factor [-];

$V_{p}$ pore volume $\left[\mathrm{m}^{3}\right]$;

$V_{\text {lens }}$ lens volume $\left[\mathrm{m}^{3}\right]$;

$X, Y, Z$ coordinate system axes [-];

$P_{1}, P_{2}$ pore intersection points [-];

$h$ height $[\mathrm{m}]$;

$d$ distance between two spheres [m];

$2 a$ unit cell size $[\mathrm{m}]$; 
$r_{z} \quad$ in-plane pore radius [m];

$t$ normalized thickness [-];

$x$ integration parameter [m];

$z \quad$ heat flux direction $[\mathrm{m}]$;

$\varphi$ Fraction defined in Eq. 6.

\section{Introduction}

Carbon foams are cellular structures that consist of randomly distributed spherical pores. The size of the pores of a typical carbon foam is 100 to 500 microns. Due to its complex structure of three-dimensional interconnected pores, carbon foams are very difficult to model analytically. Several researchers studied the effective conductivity of foams. Calmidi [1998] considered the structure of the metal foam to be made of dodecahedron-like cells with 12-14 pentagonal or hexagonal faces. The edges of the cells are formed by individual ligaments and it is considered that there is a lumping of material (intersection) at intersection points of the ligaments. This approach was successfully used by Kunny [1960], Zehner [1970], Hsu [1994], and Hsu [1995] to study the effective thermal conductivity of packed beds.

Fu et al [1998] developed an analytical model to determine the effective thermal conductivity of cellular ceramics. Two unit cells were developed to predict the effective thermal conductivity of porous materials using the electrical-circuit analogy. The first unit cell was a cubic-shaped box. The second unit cell was a cube with a pore in the center.

Tee et al. [1999] studied the thermal conductivity of carbon foam using a geometrical model that consisted of a unit cell made up of twelve struts with square cross-sectional area and eight cubic strut junctures. Tee et al. also used the analogy between thermal and electric resistors and simulated the unit cell by using series and parallel combinations of resistors.

Bhattacharya et al. [2002] studied the thermophysical properties of high porosity metal foams. For their study they considered a model consisting of a two-dimensional array of hexagonal cells where the struts form the sides of the hexagons. The junction was taken into account by considering a circular junction of metal at the intersection of the struts. This study showed that the effective thermal conductivity depends strongly on the porosity and the ratio of the cross-sections of the fiber and the intersection.

Balantrapu et al [2005] investigated the specific surface and effective thermal conductivity of open-cell lattice structure consisting of mutually orthogonal cylindrical ligaments used in heat exchanger applications.

This paper is based on an analytical solution for total surface area and thermal conductivity of a foam that has spherical pores [Druma, 2005]. With the approximation of local one-dimensional heat flow, it is possible to determine the thermal resistance of the bulk foam by adding the local thermal resistances. The specific surface area of the foam per unit volume is dependent on the porosity and pore size and distribution in the foam. In this study, the surface area for a foam with 100 micron pores is calculated and compared to the results given by a commercial solid modeling software package. 


\section{Theory}

Fu et al. [1998] used a representative cubic unit cell to determine an expression for the effective thermal conductivity. In this study two models are examined; the first is the simple cubic model with a hollow sphere in the center of a cube. The second model consists of a rectangular-shaped unit box (cubic) with the transverse section of the solid beams that enclose the unit cells being squares with normalized thickness $t$. Using the electrical-circuit analogy, the effective thermal conductivity of the second model can be expressed as (Fu et al. [1998]):

$$
\frac{K_{e}}{K_{g}}=\left[\frac{2 t}{(1-2 t)^{2}\left(K_{s} / K_{g}\right)+\left[1-(1-2 t)^{2}\right]^{2}}+\frac{(1-2 t)}{4 t^{2}\left(K_{s} / K_{g}\right)+\left(1-4 t^{2}\right)}\right]^{-1}
$$

To use Eq. (1) above, only one geometrical parameter is needed and that is the normalized thickness of the strand or ligament, $t$. This thickness can be determined by knowing the porosity of the foam and it is given by the following equation ( $\mathrm{Fu}$ et al. [1998]):

$$
(1-2 t)^{3}+6(1-2 t)^{2} t=\frac{P}{100}
$$

Tee at al. [1999] used a similar model to study the effect of anisotropy of the carbon foam struts on the bulk thermal conductivity of the foam. The effective thermal conductivity, $K_{e}$, was calculated to be:

$$
K_{e}=K_{g}(1-t)^{2}+\frac{K_{s l} K_{s j} t^{2}}{K_{s l} t+K_{s j}(1-t)}+\frac{2 K_{s t} K_{g} t(1-t)}{K_{g} t+K_{s t}(1-t)}
$$

where $t=\frac{1}{2}+\cos \left(\frac{1}{3} \cos ^{-1}\left(\frac{2 P}{100}-1\right)+\frac{4}{3} \pi\right)$

$K_{s l}, K_{s j}$ are the thermal conductivities of the struts' longitudinal direction and of the juncture respectively [W/mK].

In the hollow-sphere-in-cube model developed by Fu et al. [1998], the porosity is given by the following:

$P=\left[2 \pi\left(-\frac{4}{3} R_{p}^{3}+\frac{3}{2} R_{p}^{2}-\frac{1}{8}\right)\right] \times 100$

The above model is valid for porosity between $52 \%$ and $96 \%$. The bulk thermal conductivity of the foam is then calculated with the following equation (Fu et al. [1998]):

$$
K_{b}=\left[\int_{0}^{b} \frac{1}{\left(A_{s} / A\right) K_{s}+\left(A_{g} / A\right) K_{g}} \frac{d x}{2 a}\right]^{-1}
$$

Based on the approach used by Bhattacharya et al. [2002], where the thermal conductivity is the square root of the sum of the squares of the parallel and series 
arrangements, Sullins et al. [2001] found the effective thermal conductivity as a function of thermal conductivity of the gas $\left(K_{g}\right)$ and material of the foam $\left(K_{s}\right)$ to be:

$$
K_{e}=\varphi\left[P \cdot K_{g}+(1-P) \cdot F \cdot K_{s}\right]+(1-\varphi) \cdot\left[\frac{F \cdot K_{s} \cdot K_{g}}{P \cdot F \cdot K_{s}+(1-P) \cdot K_{g}}\right]
$$

where: $\varphi$ is the fraction of heat transfer in parallel mode and $(1-\varphi)$ is the fraction of heat transfer in series mode.

In Eq. (6) the solid conduction efficiency factor $(F)$ accounts for the tortuous path for conduction through the cell walls.

Most of the above approaches are not applicable for the carbon foam geometry because the foam does not have well defined struts. Several authors, including Bauer [1993], have presented semi-analytical approaches using a 'pore conduction' factor that must be evaluated experimentally.

The present work modifies Fu's approach to develop an analytical model for the thermal conductivity of a foam with spherical pores that may be closed pores or interconnected (overlapping) pores. In this approach, the local thermal resistances are summed up, or integrated to determine the resistance of the bulk foam. The focus of this study is to develop analytical solutions for total surface area and thermal conductivity of carbon foams based on unit cells, where the pores are distributed in a body centered cubic cell pattern. Such a unit cell is more representative of carbon foams than the cubic structure built of straight beams that has been used by Tee et al. [1999].

\section{Determination of Surface area of the foam}

The surface area of the foams is of particular interest in heat transfer applications where the main heat removal mechanism is convection. The open cell foams are therefore the main interest of this study. The foam will be modeled with pores arranged in a body centered cubic (BCC) cell distribution. In this model, the open cell foams have open cells (interconnected porosity) when the porosity $\mathrm{P}$ is greater than 68\% $(P>68 \%)$. Above this porosity the pores having the diameter, $D_{p}<\frac{2 a \sqrt{3}}{2}$, become interconnected.

The approach follows the mathematical model developed by Druma [2005] by considering a coordinate system with the $\boldsymbol{O} \boldsymbol{X}$ axis oriented in the direction of the main diagonal of the unit cell cube and the origin in the center point of the middle sphere (as shown in Fig.1). The equations of the two spherical pores (center and corner pore) can be written as (Druma [2005]):

$$
\begin{aligned}
& X^{2}+Y^{2}+Z^{2}=R_{p}^{2} \\
& (X-d)^{2}+Y^{2}+Z^{2}=R_{p}^{2}
\end{aligned}
$$

where $d=\frac{2 a \sqrt{3}}{2}$ is the distance between the two spheres (half diagonal of the unit cell). 


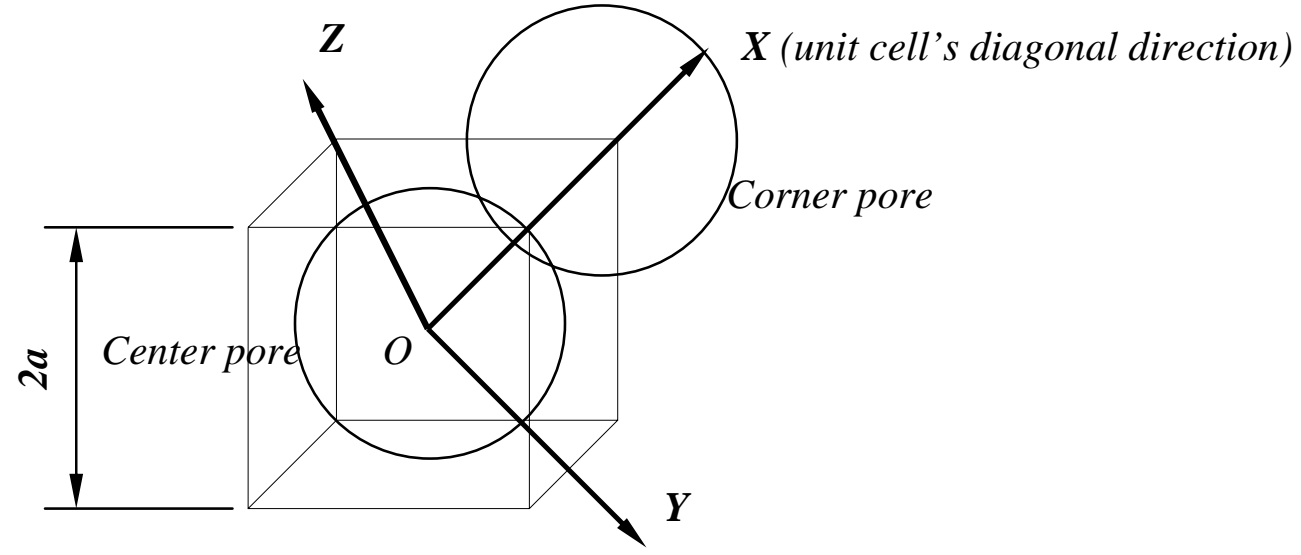

Figure 1: Spatial representation of the $O x$ axis and pore intersection (Druma [2005])

Combining the two equations yields:

$X=\frac{d}{2}$

Equation (7.a) shows that the "spherical pores intersect at the mid-distance between the two centers and the intersection curve has the equation $Y^{2}+Z^{2}=R_{p}^{2}-X^{2} \rightarrow$ $Y^{2}+Z^{2}=R_{p}^{2}-\frac{d^{2}}{4}$ which is a circle in the plane perpendicular on the main diagonal of the cubical cell and with the radius $r_{i}=\sqrt{R_{p}^{2}-\frac{d^{2}}{4}}$ ” (Druma [2005]) as shown in Fig. 2.

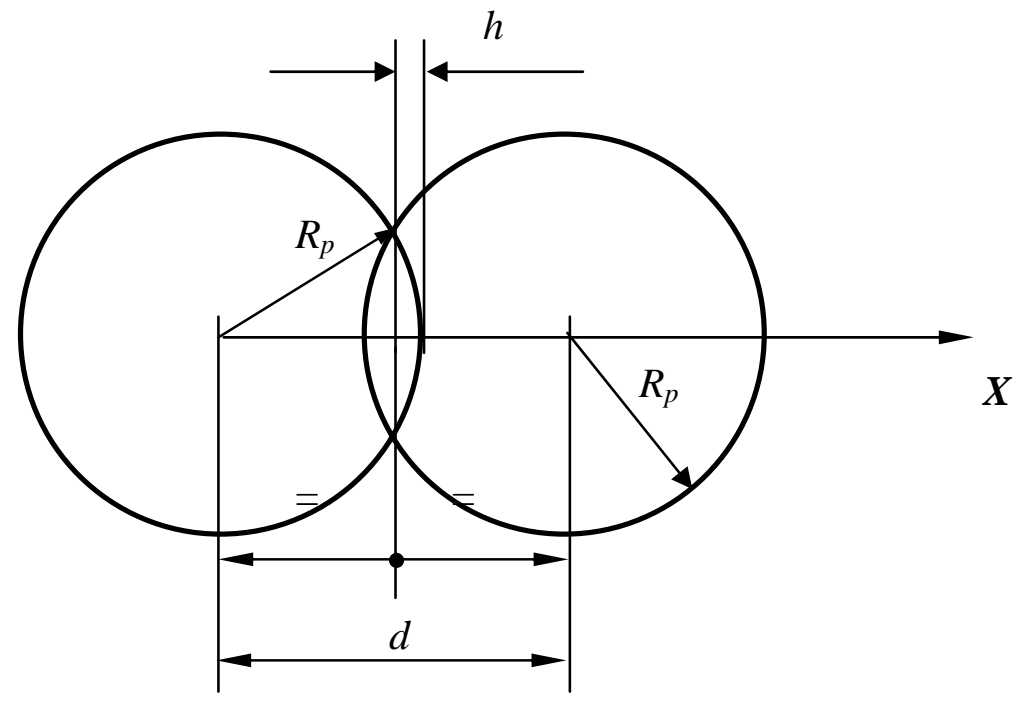

Figure 2: Planar section of the pores intersection (Druma [2005]) 
It can be concluded that the intersection of the two pores is a "three-dimensional lens" and its volume can be found by adding the volumes of the two spherical caps forming the lens.

For the open-cell foams, the void volume can be calculated with the following (Druma [2005]):

$V_{p}=\frac{8 \pi R_{p}^{3}}{3}-8 V_{\text {lens }}$

where

$V_{\text {lens }}=\frac{2 \pi h^{2}}{3}\left(3 R_{p}-h\right)$

with $h=R_{p}-\frac{d}{2}$ is the height of the intersection lens [m]

Therefore $V_{\text {lens }}=\frac{\pi}{12}\left(4 R_{p}+d\right)(2 R-d)^{2}$ and the porous volume yields:

$V_{p}=\frac{2 \pi}{3}\left(12 R_{p}^{2} d-12 R_{p}^{3}-d^{3}\right)$

The porosity of the unit cell is therefore:

$P=\left[\frac{\frac{2 \pi}{3}\left(12 R_{p}^{2} d-12 R_{p}^{3}-d^{3}\right)}{8 a^{3}}\right] \times 100$

Replacing $d=\frac{2 a \sqrt{3}}{2}$ into Eq. 11 and rearranging the resulting equation yields:

$8 a^{3}\left(\frac{\pi \sqrt{3}}{4}+\frac{P}{100}\right)-2 \pi D_{p}^{2} \sqrt{3} a+\pi D_{p}^{3}=0$

Equation 12 can be used to find the size of the unit cell for a given porosity and pore diameter.

The constraint imposed to the solution is:

$D_{p} \leq 2 a<\frac{2 D_{p}}{\sqrt{3}}$

The restriction formulated by Eq.13 can be translated as:

$0.68<P<0.94$

The equation can be solved numerically yielding a solution that satisfies the geometrical constrain given by Eq. 13 .

Total surface area of the foam (open cell)

$S_{p}=8 \pi R_{p}^{2}-8 S_{\text {lens }}$

Total surface area of the lens is given by

$$
S_{\text {lens }}=2 S_{\text {cap }}=4 \pi \int_{R-h}^{R} \sqrt{R_{p}^{2}-x^{2}} \sqrt{1+\frac{x^{2}}{R_{p}^{2}-x^{2}}} d x=4 \pi R_{p} h=4 \pi R_{p}\left(R_{p}-\frac{d}{2}\right)
$$


Replacing Eq. 16 and $d=\frac{2 a \sqrt{3}}{2}$ into Eq. 15, the total area of the unit cell (open cell foams) can be written as:

$$
S_{p}=8 \pi R_{p}^{2}-32 \pi R_{p}\left(R_{p}-\frac{d}{2}\right)=2 \pi D_{p}\left[D_{p}-2\left(2 D_{p}-2 a \sqrt{3}\right)\right]=2 \pi D_{p}\left[4 a \sqrt{3}-3 D_{p}\right]
$$

For open cell foams with porosity above $94 \%$, the diameter of the center pore becomes larger than the unit cell and the size of the unit cell can be calculated with the following equation:

$8 a^{3}\left(\frac{\pi \sqrt{3}}{4}+\frac{\pi}{2}+\frac{P}{100}\right)-2 \pi D_{p}^{2} \sqrt{3}\left(\frac{\sqrt{3}}{2}+1\right) a+2 \pi D_{p}^{3}=0$

with the constraint that $2 a<D_{p}<\frac{3 a \sqrt{2}}{2}$ for structural integrity.

Total surface area of the unit cell, for open cell foams, with porosity above $94 \%$ can be calculated with the following formula:

$$
S_{p}=2 \pi D_{p}\left[D_{p}-4\left(D_{p}-a \sqrt{3}\right)\right]-12 \frac{\pi D_{p}}{2}\left(D_{p}-2 a\right)=2 \pi D_{p}\left[2 a \sqrt{3}(\sqrt{3}+2)-6 D_{p}\right]
$$

The results for 100 microns pore diameter and different porosities are presented in Table 1 and compared with results from a solid modeling software program (Solid Edge).

Table 1: Surface areas (unit cell) for open and closed cell foams

\begin{tabular}{|c|c|c|c|}
\hline \multirow{2}{*}{$\begin{array}{c}\text { Porosity } \\
{[\%]}\end{array}$} & \multicolumn{2}{|c|}{ Surface area $\left[\mathrm{mm}^{2}\right]$} & $\begin{array}{c}\text { Percentage } \\
\text { Difference }\end{array}$ \\
\cline { 2 - 4 } & Analytical & $\begin{array}{c}\text { Software } \\
\text { (Solid Edge) }\end{array}$ & {$[\%]$} \\
\hline 70 & 0.06039 & 0.06029 & 0.166 \\
\hline 75 & 0.05421 & 0.05419 & 0.037 \\
\hline 80 & 0.04795 & 0.04793 & 0.042 \\
\hline 85 & 0.04153 & 0.04156 & 0.072 \\
\hline 90 & 0.03482 & 0.03485 & 0.086 \\
\hline 95 & 0.0261 & 0.02614 & 0.153 \\
\hline
\end{tabular}

Table 1 shows that the surface area calculated with the analytical formulas derived above matches very well with the solid modeling software prediction (difference below $0.2 \%$ between the solid modeling software and analytical result). The formulas developed above can therefore be used to estimate the surface area for open- cell cellular foams.

\section{Determination of bulk thermal conductivity}

Based on the body cubic centered unit cell presented in Fig. 3 below, an analytical model has been developed to predict the thermal conductivity of the foam. The model is based on the thermal resistance formula (Fu et. al. [1998]) using the analogy between thermal and electrical resistance. In this model, thermal resistances for differential 
elements are summed up by integration to produce the total resistance. Conductivity is calculated as the inverse of the total thermal resistance. The inaccuracy inherent in this method is the assumption that a one-dimensional thermal resistance is valid locally, even though the cross section of the solid phase in the foam changes as a function of position in the foam.

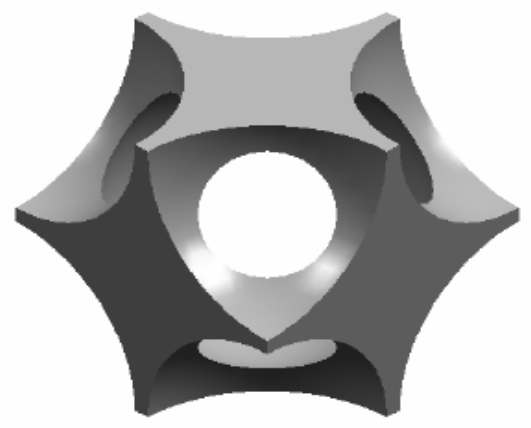

Figure 3: Spherical unit cell usea to moaeı tne porous medium (Druma [2005])

If the size of the unit cell is $2 a$, the configuration of the unit cell (symmetrical with respect to the middle pore) is shown in the Fig. 4 below:

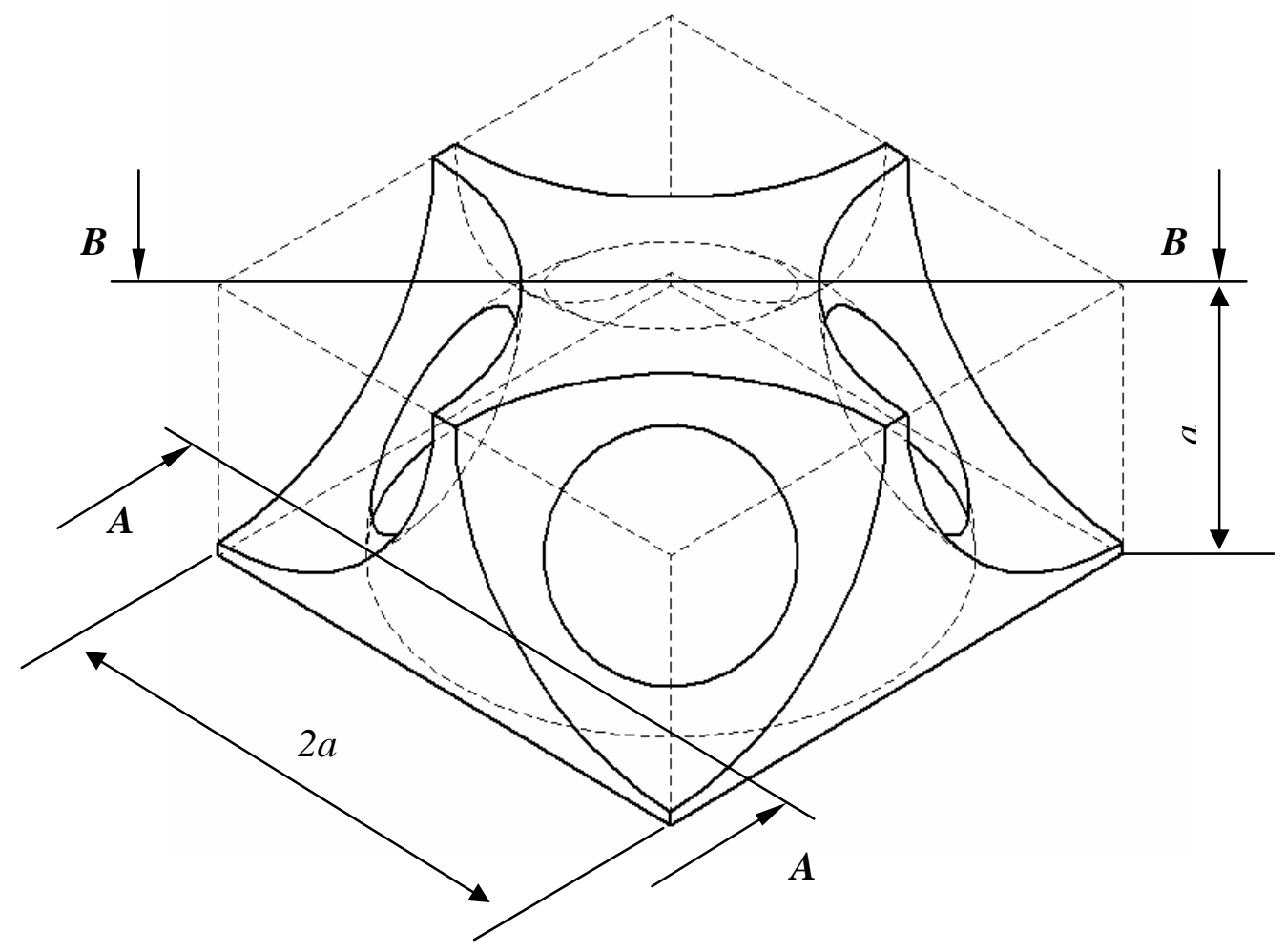

Figure 4: BCC unit cell - half model

The model is subject to the dimensional constrains given by Eqs. 14 and 15, above. 
From Fig. 4, it can be seen that the intersection between the center pore and the corner pores is circular, situated in a plane perpendicular to the diagonal of the unit cell as shown below (see Fig. 5).

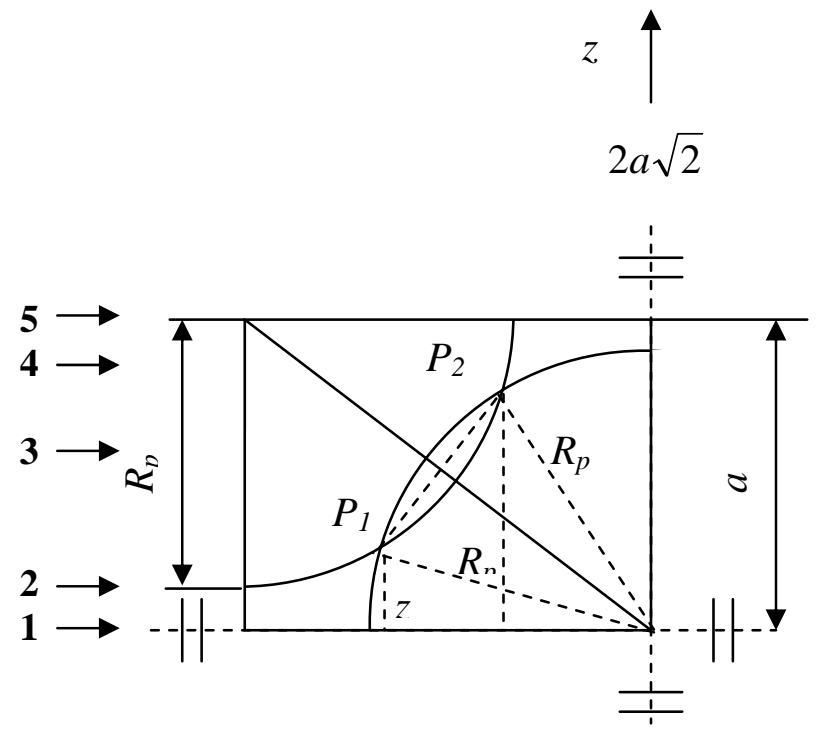

Figure 5: Section $B-B$ (quarter of the diagonal plane)

The two points where the pores become interconnected ( $P_{1}$ and $P_{2}$ in Fig. 5) are situated at the heights:

$$
\begin{aligned}
& z_{1}=\frac{a}{2}-\sqrt{\frac{2 R_{p}^{2}}{3}-\frac{a^{2}}{2}} \\
& z_{2}=\frac{a}{2}+\sqrt{\frac{2 R_{p}^{2}}{3}-\frac{a^{2}}{2}}
\end{aligned}
$$

For open cell foams (BCC case), the size of the unit cell can be calculated with the formula given by Eq. 12 .

The local radiuses of the circular pore surfaces containing the cross-section (B-B) can be calculated with the formulas:

Center pore: $r_{z}=\sqrt{R^{2}-z^{2}}$

Corner pore: $r_{z}^{\prime}=\sqrt{R^{2}-(a-z)^{2}}$

where $z$ is the vertical coordinate measured from the center of the middle pore [m].

Table 2 below contains the cross-section perpendicular to the axis of interest for calculating the effective thermal conductivity at different points in the unit cell as shown in Fig. 5. As the pore configuration changes, so does the planar cross-section. However, the total cross section area is constant: $A=4 a^{2}$. 
Table 2: Cross-sections through the unit cell and void area (Druma, [2005]).

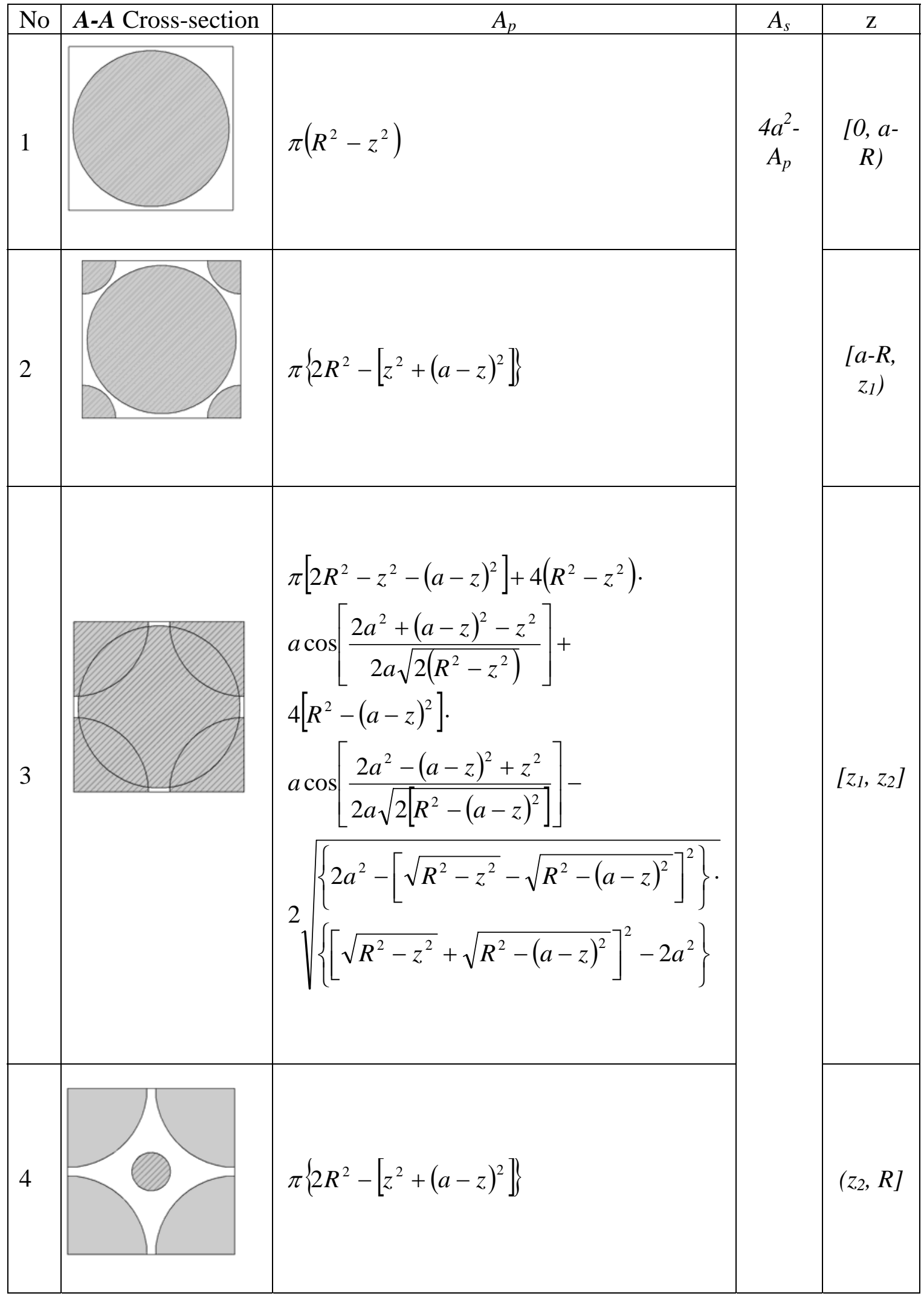




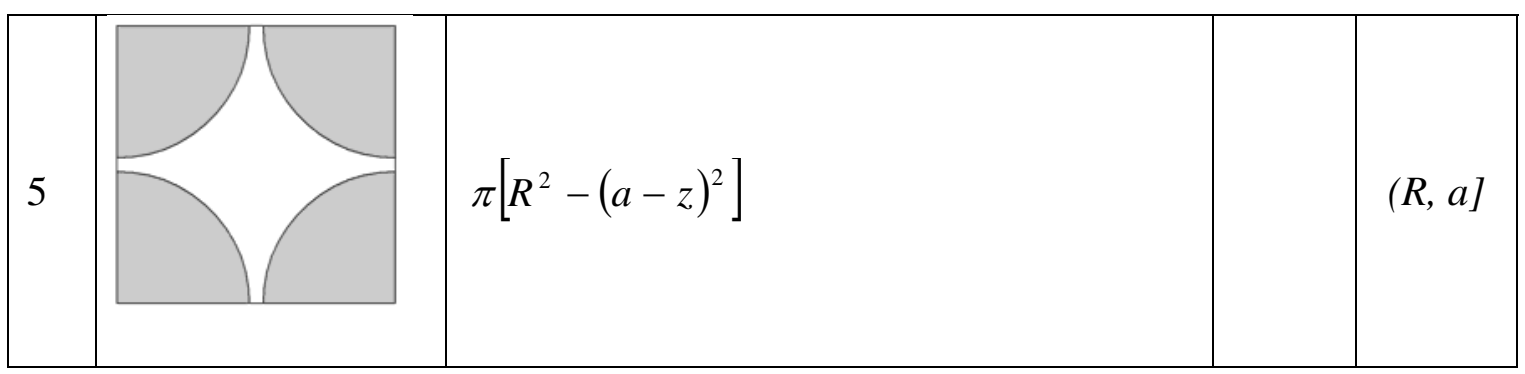

Using the definition of the thermal resistance, the bulk thermal conductivity can be calculated from the following formula:

$$
K_{b}=\frac{a}{R_{t} A}
$$

For a finite increment $d z$ along the vertical axis, the thermal resistance $d R_{t}$ is:

$d R_{t}=\frac{d z}{A_{s} K_{s}+A_{g} K_{g}}$

Integrating the equation above between 0 and $a$, and replacing the thermal resistance in Eq. 24 yields the bulk thermal conductivity:

$$
K_{b}=\frac{a}{A R_{t}}=\frac{1}{\int_{0}^{a} \frac{A}{A_{s} K_{s}+A_{g} K_{g}} \frac{d z}{a}}
$$

For foams, the thermal conductivity of the pore's content (gas) is much lower than the solid thermal conductivity and can therefore be approximated to zero. In this case, the formula above becomes:

$$
K_{b}=\frac{a}{A R_{t}}=\frac{1}{\int_{0}^{a} \frac{A}{A_{s} K_{s}} \frac{d z}{a}}
$$

The effective thermal conductivity of the foam with constant solid thermal conductivity can therefore be calculated as follows:

$$
K_{e}=\frac{K_{b}}{K_{s}}=\frac{1}{\int_{0}^{a} \frac{A}{A_{s}} \frac{d z}{a}}
$$

The integral in Eq. 28 does not have an analytical solution and is calculated numerically using an adaptive quadrature and the results are presented in Table 3 [Druma, 2005] below. Table 3 also shows the comparison between the finite element (FEM) calculation carried out [Druma et al., 2004] by using a commercial software 
program (ALGOR), and the semi-analytical model developed by Bauer [1993]. Bauer developed a semi-analytical approach to obtain the following equation:

$$
K_{e}=\frac{K_{b}}{K_{s}}=\left(1-\frac{P}{100}\right)^{1 / n}
$$

where ' $n$ ' is an unknown parameter, usually termed the 'pore conduction factor'. A value of ' $n=0.77$ ' provides a good fit to some of the experimental data analyzed by Bauer, and is also comparable to numerical results [Druma et al., 2004]. It can be observed that the analytical model overpredicts the thermal conductivity; this is to be expected since the analytical model does not take the tortuosity of the heat transfer path into account.

Table 3: Effective Thermal Conductivity: Analytical - FEM comparison

\begin{tabular}{|c|c|c|c|c|c|}
\hline \multirow[b]{2}{*}{ No. } & \multirow[b]{2}{*}{$\begin{array}{c}\text { Porosity } \\
{[\%]}\end{array}$} & \multirow[b]{2}{*}{$\begin{array}{c}2 \mathrm{a} \\
{[\mathrm{mm}]}\end{array}$} & \multicolumn{3}{|c|}{$\mathrm{K}_{\mathrm{e}}$} \\
\hline & & & $\begin{array}{c}\text { Analytical } \\
\text { Model }\end{array}$ & FEM & $\begin{array}{l}\text { Bauer } \\
\text { Model }\end{array}$ \\
\hline 1 & 70 & 0.114 & 0.27 & 0.251 & 0.21 \\
\hline 2 & 75 & 0.111 & 0.213 & 0.197 & 0.165 \\
\hline 3 & 80 & 0.108 & 0.159 & 0.147 & 0.124 \\
\hline 4 & 85 & 0.105 & 0.111 & 0.1 & 0.085 \\
\hline 5 & 90 & 0.102 & 0.077 & 0.067 & 0.05 \\
\hline
\end{tabular}

However, the analytical model matches the FEM results better than the Bauer model, and this can be seen in Figure 6. The comparison between the FEM and anaytical model is also shown in Fig. 6, along with Bauer model (Bauer [1993]), Cubic model (Fu et.al. [1998]), and Hollow-Sphere-in-Cube Model (Fu et.al. [1998]) . It should be noted that the pore diameter is kept constant at 100 micrometers for all porosities. Therefore, as the porosity increases, the distance between pores becomes smaller. In other words, the variable porosity at constant pore size causes the change in the size of the unit cell. The rule of mixtures assumes a linear relationship with the contribution of each phase according to volume fractions and provides the highest estimate of the conductivity value; it is equivalent to using $\varphi=0$ in Eq. 6 . 


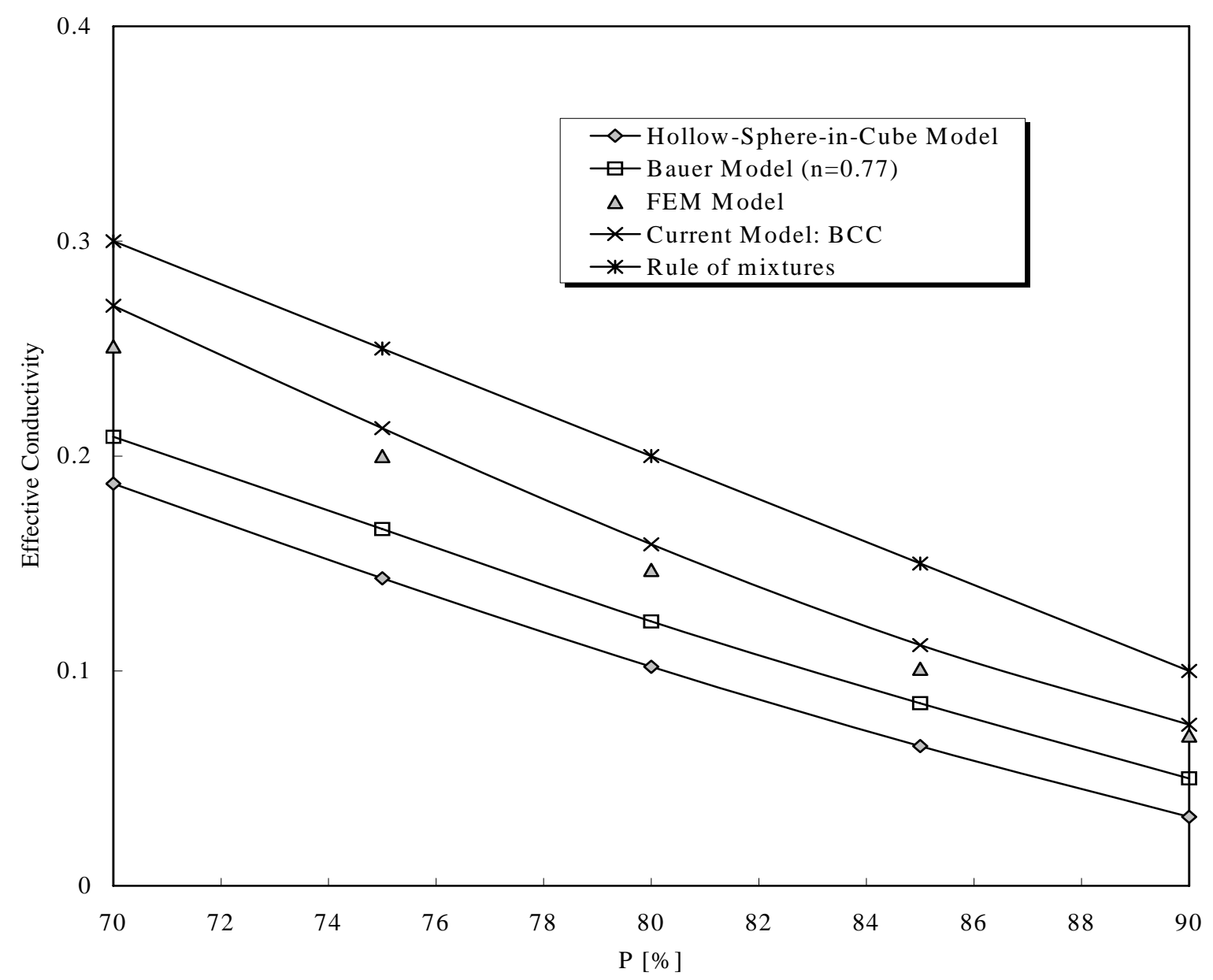

Figure 6: Open cell thermal conductivity - comparison between analytical and FEM results (Druma [2005])

\section{Conclusions}

An analytical model has been developed to determine the surface area and thermal conductivity of foams with spherical pores and different levels of porosity. The results are compared with solutions from a numerical calculation of a commercial software program. It has been shown that the analytical solution produces accurate results for the surface area. The thermal conductivity results are comparable to the FEM results but overpredict the FEM results because of the one-dimensional approximation of the heat flux. It is also shown that the results depend on the assumption of the pore shape as well as the distribution of pores in the foam. 


\section{References}

Balantrapu, K., Deepty, R.S., Herald, C.M., Wirtz, R.A., Porosity, Specific Surface Area and Effective Thermal Conductivity of Anisotropic Open Cell Lattice Structures, Proceedings of IPACK2005, IPACK2005-73191, California, 2005.

Bauer, T.H., "A general analytical approach toward the thermal conductivity of porous media”, Int. Journal of Heat and Mass Transfer, vol. 36(17), 4181-4191, 1993.

Bhattacharya, A., Calmidi, V.V., and Mahajan, R.L., Thermophysical properties of high porosity metal foams, International Journal of Heat and Mass Transfer, 45 (2002) 1017-1031.

Calmidi, V.V., Transport phenomena in high porosity fibrous metal foams, Ph.D. Dissertation, University of Colorado, Boulder, Colorado, 1998.

Druma, A.D., Alam, M.K., Druma, C., Analysis of thermal conduction in carbon foams, International Journal of Thermal Sciences, vol. 43(7), pp. 689-695, 2004.

Druma, A.M., Analysis of carbon foams by finite element method, Ph.D. Dissertation, Ohio University, Athens, Ohio, 2005

Fu, X., Viskanta R., Gore, J.P., Prediction of effective thermal conductivity of cellular ceramics, Int. Comm. Heat Mass Transfer, Vol. 25, No. 2, pp. 151-160, 1998.

Hsu, C.T., and Cheng P., "Modified Zehner-Schlunder models for stagnant thermal conductivity of spatially periodic porous media, ASME Journal of Heat Transfer, 37 (17), 2751-2759, 1994.

Hsu, C.T., and Cheng P., “A lumped parameter model for stagnant thermal conductivity of spatially periodic Porous Media”, ASME Journal of Heat Transfer, 117, 264269, 1995.

Kunnii D., and Smith J.M., "Heat Transfer Characteristics of Porous Rocks", AIChE Journal, 6, 71-78, 1960.

Sullins, A.D., and Daryabeigi, , K, "Effective Thermal Conductivity of High Porosity Open Cell Nickel Foam”, AIAA 2001-2819, 35 ${ }^{\text {th }}$ AIAA Thermophysics conference, Anaheim California, June 2001.

Tee, C.C., Klett, J.W., Stinton, D.P., and Yu N., "Thermal conductivity of porous carbon foam”, Proceedings of the 24th Biennial Conference on Carbon, July 11-16, Charleston, SC, 130, 1999.

Zhener P., and Schlunder, E.U., "Thermal Conductivity of Granular Materials at Moderate Temperatures”, Chemie. Ingr-Tech., 42, 933-941, 1970. 